\title{
Género, motivaciones y frenos en el consumo de música en directo
}

\author{
Gender, motives and barriers in live music \\ consumption
}

\author{
Manuel Cuadrado-García \\ María José Miquel-Romero \\ Juan D. Montoro-Pons \\ Universitat de València
}

\section{Resumen}

La asistencia a conciertos y festivales de música en directo ha evolucionado notablemente en los últimos tiempos consecuencia de los cambios acaecidos en este sector. La literatura muestra estudios centrados en analizar los principales determinantes de esta participación. Si bien algunos de estos trabajos incluyen el género como variable demográfica explicativa de este consumo, los resultados obtenidos son contradictorios. Por ello, el presente artículo desarrolla una investigación empírica mediante encuesta personal con el objetivo de analizar el rol del género, así como de los motivos y frenos de consumo, en la asistencia a conciertos de música. La no existencia de grandes diferencias entre hombres y mujeres se encuentra entre los principales resultados.

Palabras clave: música en vivo, conciertos, festivales, género, investigación descriptiva, marketing.

Clasificación JEL: Z11, M31.

\begin{abstract}
Attendance at live music concerts and festivals has dramatically evolved in recent times as a result of changes in this sector. The literature shows studies focused on analyzing the main determinants of this participation. Although some of these papers include gender as a demographic variable explaining consumption, results are inconclusive. Thus, the present work develops an empirical research by means of a personal survey with the aim of studying the role of gender, as well as motives and barriers of consumption, in attending music concerts. The non-existence of great differences between men and women is one of the main results.
\end{abstract}

Keywords: live music, concerts, festivals, gender, descriptive research, marketing.

JEL classification: Z11, M31. 


\section{Introducción}

El gran avance de la tecnología en los últimos años ha conllevado abrumadores cambios en la producción, distribución y consumo de música. Por lo que respecta a la industria de la música grabada se observa un declive en la venta de unidades físicas, que ha sido parcialmente compensado con un crecimiento de la compra de música en formato digital y el acceso a plataformas streaming (SGAE, 2018). La industria de la música en directo también ha experimentado una importante transformación. En este sentido, se ha consolidado una amplia y variada oferta de eventos en vivo que combinan en un mismo cartel artistas diversos, los denominados festivales. Estos muestran una concentración alrededor de los meses estivales, al tiempo que sirven de principal vía para la generación de ingresos por parte de artistas y bandas (Krueger, 2005). Los festivales coexisten junto a conciertos o giras de diverso tamaño con una distribución más uniforme a lo largo del resto del año, si bien la temporada en locales de aforo medio y pequeño tiende a desarrollarse fuera de los meses en los que los festivales abundan.

La música en vivo supone así una industria con un elevado volumen de negocio que genera efectos arrastre en otros sectores, como la hostelería o el turismo. Tal y como recoge el Cuadro 1, si bien tanto el número de conciertos como de espectadores ha experimentado una tendencia a la baja, el volumen de negocio (medido en términos corrientes a través de la recaudación generada) muestra un comportamiento creciente, alcanzando en 2107 cerca de 330 millones de euros. La caída anual en el número de conciertos se modera del 5,96\% en 2009 al 0,38\% en 2017, al igual que el número de espectadores, al pasar de una disminución del $8 \%$ en 2009 a una del $4,9 \%$ en 2017. No obstante, los datos agregados ocultan una dinámica diferente cuando comparamos los eventos de música popular en vivo considerando o no los macroconciertos. En este sentido, en la evolución de espectadores a estos macrofestivales se observa como la asistencia supuso el $21 \%$ de la audiencia total en 2017, comparado con solo el $5 \%$ en 2008. Esta dinámica hacia la concentración se agudiza al observar los ingresos. En 2017, más del $50 \%$ de la recaudación correspondió a macrofestivales, frente al $20 \%$ de 2008 . Ello confirma que son principalmente estos grandes festivales y las grandes giras de grupos o artistas los que movilizan gran parte del negocio de la música popular en vivo.

Si bien la dinámica descrita obedece en parte a la evolución cíclica de la economía, afectada por la gran recesión inaugurada en 2008, la observación de los datos conduce a dos conclusiones. Primera, el sector de música en vivo está inmerso en un proceso de concentración con un incremento del tamaño relativo de los macrofestivales y, por tanto, de su importancia en el sector. Segundo, y vinculado con lo anterior, los grandes eventos han capitalizado la mayor parte de los ingresos por taquilla con crecimientos de espectadores que han sido más moderados. Dicho de otro modo, los grandes eventos de música en vivo han sido capaces de aumentar su volumen de negocio vía aumento de precios. 


\section{CUADRO 1}

EVOLUCIÓN DE LA MÚSICA POPULAR EN VIVO EN ESPAÑA

\begin{tabular}{|c|c|c|c|c|c|c|c|}
\hline \multicolumn{4}{|c|}{ Todos los conciertos } & \multicolumn{2}{c|}{$\begin{array}{c}\text { Excluyendo } \\
\text { macrofestivales }\end{array}$} & \multicolumn{2}{c|}{$\begin{array}{c}\text { Ratios para } \\
\text { macrofestivales }\end{array}$} \\
\hline Año & Conciertos & $\begin{array}{c}\text { Especta- } \\
\text { dores }\end{array}$ & $\begin{array}{c}\text { Recauda- } \\
\text { ción }(€)\end{array}$ & $\begin{array}{c}\text { Especta- } \\
\text { dores }\end{array}$ & $\begin{array}{c}\text { Recauda- } \\
\text { ción }(€)\end{array}$ & $\begin{array}{c}\text { \% espec- } \\
\text { tadores }\end{array}$ & $\begin{array}{c}\text { \% recau- } \\
\text { dación }\end{array}$ \\
\hline 2008 & 138.613 & 35.672 .610 & 206.016 .960 & 33.813 .012 & 163.956 .320 & 5,21 & 20,42 \\
\hline 2009 & 130.346 & 32.800 .090 & 197.961 .132 & 31.025 .074 & 169.911 .231 & 5,41 & 14,17 \\
\hline 2010 & 126.907 & 31.367 .681 & 210.654 .359 & 29.263 .365 & 167.709 .415 & 6,71 & 20,39 \\
\hline 2011 & 121.722 & 28.554 .650 & 208.931 .551 & 26.140 .090 & 167.387 .158 & 8,46 & 19,88 \\
\hline 2012 & 116.446 & 27.659 .939 & 212.155 .551 & 25.040 .027 & 162.410 .889 & 9,47 & 23,45 \\
\hline 2013 & 103.208 & 24.940 .851 & 202.148 .921 & 22.111 .950 & 143.730 .688 & 11,34 & 28,90 \\
\hline 2014 & 94.643 & 24.827 .201 & 229.798 .273 & 21.139 .304 & 146.968 .213 & 14,85 & 36,04 \\
\hline 2015 & 90.212 & 24.791 .191 & 254.673 .914 & 20.424 .009 & 148.088 .908 & 17,62 & 41,85 \\
\hline 2016 & 88.259 & 25.360 .739 & 297.619 .290 & 20.127 .136 & 149.183 .412 & 20,64 & 49,87 \\
\hline 2017 & 87.924 & 26.603 .812 & 328.889 .273 & 20.927 .049 & 155.786 .663 & 21,34 & 52,63 \\
\hline
\end{tabular}

FUENTE: SGAE (2018) y elaboración propia.

El análisis geográfico de los datos pone además de manifiesto la dinámica espacial que hay detrás de ellos. En concreto, los datos de SGAE (2018) muestran como en la Comunitat Valenciana, el área geográfica donde se desarrolla el trabajo de campo de la investigación empírica de este estudio, el número de espectadores ha crecido de forma sostenida entre 2008 y 2017, pasando de 1,9 millones a cerca de 2,4 millones. Esto ha supuesto un crecimiento del porcentaje de asistencia sobre el total del territorio del Estado del $7 \%$ en 2008 al 8,7\% en 2017, siendo solo superado por Andalucía (19\%), Cataluña (17\%) y Madrid (15,8\%). Además, en cuanto a ingresos, la Comunitat Valenciana representa el 14,8\% del volumen total de negocio, también en cuarto lugar, lo que muestra la apuesta que se ha hecho en el territorio para la producción de macrofestivales. En definitiva, los datos constatan el dinamismo de la industria de la música en vivo en esta comunidad autónoma.

A pesar de la relevancia que el sector tiene, tanto en volumen de negocio como por proyección del territorio, y los importantes efectos arrastre que se extienden a sectores anejos como el turismo, los datos analizados ocultan la existencia de una industria con una cadena de valor de gran complejidad en la que intervienen multitud de agentes: artistas, agentes, promotores, agencias de booking, de contratación, empresas de ticketing, festivales, técnicos, productores, Administraciones públicas, otros organismos, y público. A este respecto, existen importantes carencias en torno a la industria de la música en vivo y su verdadero impacto. Así, no existe una base de datos completa de tal oferta, ni tampoco estudios con suficiente rigor científico que analicen la relevancia o efectos que los mismos generan a todos los niveles: artísti- 
cos, sociales, económicos y tecnológicos. La poca información existente la proporciona la Asociación de Promotores Musicales de España (APM), que representa el $80 \%$ de los conciertos de iniciativa privada que se realizan en este país, estando integrada por 64 empresas especializadas en la música en vivo. Son empresas dedicadas tanto a la gestión de grupos de música en gira como a la organización de festivales. Desde hace seis años elaboran el Anuario de la APM, una radiografía del sector de la música en vivo en nuestro país, si bien su finalidad es fundamentalmente descriptiva.

Una de las cuestiones sobre la cual hay una carencia de análisis rigurosos, es la del sesgo de género en la música en vivo. Así la última oleada de la Encuesta de Hábitos y Prácticas Culturales 2018-2019 (EHPC, Ministerio de Cultura y Deporte, 2019) muestra que, si bien el grado de interés por conciertos de música actual es ligeramente mayor en el caso de las mujeres, 6,8 sobre 10 frente a 6,5 de los hombres, el porcentaje de personas que asistieron a espectáculos de este tipo de música en el último año es dos puntos más elevado para los hombres, $31,6 \%$ frente al 28,7\% de las mujeres. Unos datos que se confirman cuando se considera el asistir una vez en los tres últimos meses, algo por encima también en el caso de los varones (Cuadro 2). Sin embargo, la situación se revierte en la asistencia a conciertos de música clásica, donde las mujeres presentan valores algo superiores a los hombres. La información anterior puede complementarse con la explicación de los motivos por los que los individuos no van más a conciertos de música. Según la mencionada encuesta, las mujeres señalan algo más el precio, y la dificultad de salir de casa como barreras a la asistencia, mientras que para los hombres tiene algo más de peso la falta de tiempo, así como de interés. Con todo, el consumo global de música en directo es algo superior en el caso de los hombres.

En este contexto, la existencia de estudios académicos específicamente centrados en la industria de la música en vivo desde una perspectiva económica, de gestión y consumo es escasa. Existen ciertos trabajos que han analizado el consumo de música popular en vivo (Montoro-Pons y Cuadrado-García, 2011) así como sus determinantes de participación y frecuencia de asistencia (Montoro-Pons y Cuadrado-García,

\section{CUADRO 2}

PREFERENCIA Y ASISTENCIA A CONCIERTOS DE MÚSICA ACTUAL SEGÚN GÉNERO

\begin{tabular}{|l|c|c|c|}
\hline & $\begin{array}{c}\text { Preferencia } \\
\text { (sobre 10) }\end{array}$ & $\begin{array}{c}\text { Porcentaje asistencia } \\
\text { último año }\end{array}$ & $\begin{array}{c}\text { Porcentaje asistencia } \\
\text { últimos 3 meses }\end{array}$ \\
\hline Hombre & 6,5 & 31,6 & 16,7 \\
\hline Mujer & 6,8 & 28,7 & 15,4 \\
\hline Total & 6,6 & 30,1 & 16,0 \\
\hline
\end{tabular}

FUENTE: EHPC 2018-2019 (Ministerio de Cultura y Deporte, 2019). 
2016). Otros se han centrado en estudiar la naturaleza y alcance de los festivales (Colomer y Carreño, 2011; Getz, Andersson y Larson, 2007; Getx, 2010; Richards, 2007), en describir y analizar algunas de sus pautas de gestión (Andersson y Carslen, 2011; Carreño, 2015) y los menos en estudiar la eficacia de alguna de sus medidas (Getz y Frisby, 1998).

El presente estudio se desarrolla con el objetivo de ahondar en el conocimiento del comportamiento del consumidor en el contexto de la música en directo y poder aportar ciertos resultados y contenidos a la literatura. Concretamente, pretendemos analizar la influencia del género en el consumo de prácticas culturales vinculadas con la música, y de manera específica con la música en vivo, así como su relación con las motivaciones positivas y negativas de asistencia y la disposición a pagar por la entrada de un concierto de música. Para ello, este artículo continúa con la consiguiente revisión de la literatura y sigue describiendo la investigación exploratoria desarrollada, sus principales resultados y finaliza presentando las conclusiones más relevantes.

\section{Género y consumo de música}

Cualquier disciplina científica relacionada con el comportamiento humano, la sociedad y la cultura tiene en cuenta la influencia del género (Eckert y McConnellGinet, 2003). El género ha sido, y continúa siendo, uno de los criterios de segmentación más habitualmente utilizados por su fácil identificación y acceso, así como por proporcionar segmentos de tamaño rentable (Kim, Lehto y Morrison, 2007). Sin embargo, para implementarlo de forma adecuada en una estrategia, es necesario entender en qué aspectos condiciona el comportamiento de los individuos.

En este sentido, son varias las justificaciones que ayudan a entender cómo y por qué hombres y mujeres se comportan de manera distinta (Putrevu, 2001). Una pequeña parte de sus diferencias puede atribuirse a cuestiones puramente biológicas (cromosomas sexuales, hormonas sexuales, lateralización del cerebro, etc.), mientras que la otra gran parte hay que justificarla desde otros ámbitos. Así, la investigación en el campo de la socialización apunta al concepto de identificación del rol de género. En este contexto, la Teoría del Rol Social (Eagly, 2013) sugiere que las diferencias entre hombres y mujeres en aptitudes y rasgos de personalidad a menudo reflejan los roles tradicionales de una sociedad; señalándose que los hombres se vinculan más con la asertividad, la autoeficacia y el dominio, mientras que las mujeres con la sensibilidad hacia uno mismo y hacia los demás. Paralelamente, hay constancia empírica de que cada sexo procesa la información de manera distinta: los hombres son «procesadores selectivos» que confían solo en la información disponible y los aspectos más desatacados de un mensaje, mientras que las mujeres son «procesadoras exhaustivas» que asimilan toda la información disponible antes de dar un juicio (Meyers-Levy y Maheswaran, 1991). 
Al término género se vinculan matices varios como rol de género, identidad de género o estereotipo de género. Todos ellos plantean cómo son o se comportan hombres y mujeres en una determinada cultura y entorno social (Birknerová et al., 2018). Oakley (1972), uno de los primeros en hacer uso del término género, lo delimitó como una construcción social y cultural, distinta del término sexo basado en diferencias biológicas (Krook y Childs, 2010). El género tiene que ver con «las distintas formas en las que hombres y mujeres interactúan en los encuentros sociales del día a día» (Gilmore, 1998).

La conceptualización más convencional del género se identifica con un binario fijo, hombre-mujer, asignándose a cada género unos roles sociales, unos comportamientos y, en general, unas ideas sobre lo que es correcto e incorrecto según dicho género. Ello se manifiesta en la toma de decisiones y procesos de consumo (preferencias, expectativas, criterios de decisión, etc.) (Suhányi y Suhányiová, 2014). En definitiva, tal y como manifiesta Connell (2002), el género juega un papel clave en la vida personal del individuo, en sus relaciones sociales y en la cultura, pero al mismo tiempo trae consigo ciertos prejuicios, ideologías y mitos que le condicionan.

Las connotaciones de género van transmitiéndose y reproduciéndose a través del proceso de aprendizaje social; sin embargo, estas van cambiando con el tiempo (Cviková, Debrecínová y Kobová, 2007), por lo que es clave entender en qué aspectos hombres y mujeres siguen, o no, teniendo diferentes comportamientos y procesos de consumo.

\subsection{Género y música}

En el ámbito de la música, los trabajos que analizan la influencia del género los podemos clasificar en dos grandes grupos: aquellos que estudian su influencia en la etapa de pre-consumo, centrados en conocer su repercusión sobre gustos, actitudes, motivaciones o frenos, y los que analizan su influencia en el propio consumo o participación.

Dentro del primer grupo cabe mencionar investigaciones como la de Van Eijck (2001), quien constató que: la música clásica es más apreciada por las mujeres; el consumidor omnívoro es mayoritariamente hombre; y el género no juega ningún papel en la segmentación de los individuos según sus gustos musicales. Asimismo, las mujeres prestan significativamente más atención que los hombres a la música de los anuncios (Birknerová et al., 2018). El género, sin embargo, no resultó determinante en el estudio de Prieto-Rodríguez y Fernández-Blanco (2000) sobre las preferencias por la música clásica o popular.

De manera más concreta, Webster y Weir (2005) analizaron la influencia del género en la percepción que la audiencia tiene de lo que la música transmite, concluyendo que los hombres tienen una percepción más alegre de la música que las mujeres. No obstante, los autores apuntaron la importancia de considerar además el ritmo de la canción, al poder este matizar las diferencias entre géneros. De manera 
similar, Kellaris y Rice (1993) consideraron, mucho antes, que la reacción a la música según el género estaba condicionada por el nivel de sonido, al comprobar que las mujeres reaccionan peor que los hombres cuando el volumen de la música es alto. Además, Hu y Lee $(2012,2016)$ y Lee y Hu (2014) resaltaron que las diferencias culturales entre países también determinan que hombres y mujeres tengan distintas percepciones.

Otros estudios analizan la influencia del género en las motivaciones a participar en actividades vinculadas con la música. Así, Liao y Tsai (2018) mostraron que, aun no habiendo diferencias por género en las motivaciones a participar, existen diferencias en cuanto a los frenos: las restricciones familiares y económicas juegan un mayor peso en el caso de las mujeres que en los hombres. En el caso más específico de asistencia a conciertos de música clásica, Chen y Hu (2006) concluyeron que para los hombres, es la falta de interés por tales conciertos y la forma en la que se anuncian lo que les frena a ir, mientras que para las mujeres es la atmósfera demasiado seria que generan tales eventos.

El segundo grupo de trabajos estudia la influencia del género sobre el comportamiento de consumo de música o actividades vinculadas con la música. Entre estas investigaciones Kinnally et al. (2008) analizaron el comportamiento de descarga de música no autorizada, concluyendo que, si bien el consumo de música descargada era menor en los hombres, las actitudes hacia la descarga de música eran las mismas para ambos géneros. En este mismo contexto de música online, Nel, Raubanheimer y Bounagui (2009) pudieron comprobar que la confianza percibida y la autoeficacia percibida tenían mayor influencia en la intención de compra en el caso de las mujeres que en los hombres, mientras que ocurría lo contrario en relación a la influencia de la diversión percibida.

En la misma línea, Montoro-Pons y Cuadrado-García (2011) demostraron que el género es relevante en el consumo de música popular, tanto pregrabada como en directo, al disminuir la probabilidad de participación femenina en ambos tipos de consumo, aunque en mayor medida en el caso de la asistencia a conciertos. Asimismo Chen y $\mathrm{Hu}$ (2006) señalaron que las estudiantes universitarias asistían más a conciertos de música clásica que sus compañeros hombres, tal y como también concluyeron Favaro y Frateschi (2007) para conciertos de música en general, al apuntar que ser mujer, junto a otras características, es un buen predictor de tal comportamiento.

Con todo, podemos concluir que, si bien existen trabajos al respecto, estos son muy diversos y centrados en aspectos muy variados, no pudiendo derivarse una conclusión clara y sólida sobre cómo el género determina dicho proceso de consumo y, de manera específica, las motivaciones y frenos a la participación. 


\section{Investigación: objetivos y metodología}

\subsection{Objetivos}

El objetivo de este trabajo consiste en analizar la influencia del género en la asistencia a conciertos de música y su relación con los motivos y frenos de la misma asistencia, contribuyendo de esta manera a la literatura sobre género y participación en la música en vivo.

De manera específica la investigación persigue dar respuesta a las siguientes cuestiones a investigar:

1. Conocer la existencia de diferencias significativas entre hombres y mujeres en la frecuencia de prácticas culturales directamente vinculadas con la música.

2. Analizar los principales motivos y frenos de asistencia a conciertos en función del género.

3. Profundizar de manera específica en el conocimiento de la predisposición a pagar por asistir a conciertos y conocer las posibles diferencias según género.

\subsection{Metodología}

Con el propósito de dar respuesta al objetivo planteado se llevó a cabo un estudio de carácter descriptivo, definiendo el universo como individuos mayores de 18 años que hubieran escuchado música, al menos una vez, en el último mes. El método de muestreo empleado fue no probabilístico por cuotas en base a la edad y al género del individuo, obteniendo una muestra final de 753 entrevistados.

La técnica de recogida de información fue la entrevista personal, para lo cual se diseñó un cuestionario estructurado, organizado en tres bloques. El primero de ellos, recogía información sobre prácticas y actividades culturales en general; el segundo se centraba en la música en vivo, identificando motivaciones y frenos de asistencia, así como el precio dispuesto a pagar, y finalmente, el último bloque permitía identificar el perfil sociodemográfico del entrevistado. La gran mayoría de preguntas utilizadas en el cuestionario eran escalas métricas, tipo Likert, de cinco puntos (1.-Total desacuerdo a 5.-Total acuerdo). Mención especial requiere la pregunta relativa a la frecuencia de realización de actividades vinculadas con la música. La misma se planteó con tres alternativas de respuesta genéricas (nunca, ocasionalmente y frecuentemente) ante la alta variabilidad en la repetición de las diferentes prácticas (por ejemplo, descargar música frente a asistir a conciertos) y la imposibilidad de establecer intervalos comunes para cada una de ellas. El trabajo de campo se realizó en diciembre de 2018. Los datos fueron analizados de manera univariante y multivariante a través del paquete informático SPSS 24. En el Cuadro 3 se muestra una síntesis del perfil sociodemográfico de la muestra del estudio. 


\section{CUADRO 3 \\ PERFIL SOCIODEMOGRÁFICO DE LA MUESTRA}

(En \%)

\begin{tabular}{|l|l|r|}
\hline \multirow{4}{*}{ Género } & Hombre & 50,3 \\
\cline { 2 - 3 } Edad media & Mujer & 49,7 \\
\hline \multirow{5}{*}{ Nivel de estudios } & \multicolumn{2}{|c|}{38,9 años } \\
\hline \multirow{5}{*}{ Ocupación principal } & Sin estudios & 2,0 \\
\cline { 2 - 3 } & Primaria/Secundaria & 21,0 \\
\cline { 2 - 3 } & Bachillerato/Ciclos & 31,2 \\
\cline { 2 - 3 } & Universidad & 45,8 \\
\hline \multirow{5}{*}{ Situación familiar } & Estudiante & 25,5 \\
\cline { 2 - 3 } & Empleado por cuenta propia & 14,3 \\
\cline { 2 - 3 } & Empleado por cuenta ajena & 48,5 \\
\cline { 2 - 3 } & Desempleado & 3,6 \\
\cline { 2 - 3 } & Tareas domésticas & 3,3 \\
\cline { 2 - 3 } & Jubilado & 4,8 \\
\hline \multirow{5}{*}{ Nacionalidad } & Sin pareja/sin hijos & 30,9 \\
\cline { 2 - 3 } & Sin pareja/con hijos & 5,8 \\
\cline { 2 - 3 } & Con pareja/sin hijos & 17,1 \\
\cline { 2 - 3 } & Con pareja/con hijos & 46,1 \\
\hline & Española & 43,3 \\
\cline { 2 - 3 } & Francesa & 9,3 \\
\cline { 2 - 3 } & Británica & 7,7 \\
\cline { 2 - 3 } & Italiana & 7,3 \\
\cline { 2 - 3 } & Neerlandesa & 3,3 \\
\cline { 2 - 3 } & Otra & 29,1 \\
\hline
\end{tabular}

FUENTE: Elaboración propia.

De la lectura e interpretación del cuadro se puede afirmar que la muestra está similarmente repartida entre hombres y mujeres; la edad media es de 39 años, la gran mayoría son españoles, con estudios universitarios, trabajando por cuenta ajena, con pareja y con hijos.

\section{Resultados}

\subsection{Prácticas vinculadas con la música}

Las prácticas musicales incluidas para conocer la implicación de los participantes en el estudio en términos de frecuencia fueron: descarga de música de Internet, 
escucha de música en streaming, asistencia a musicales y asistencia a conciertos en vivo. Concretamente se solicitó a los entrevistados que manifestaran su frecuencia de realización con la siguiente escala de tres puntos: nunca (1), ocasionalmente (2), y frecuentemente (3). En el Cuadro 4 se resumen los resultados.

A nivel global, para toda la muestra, se constata que escuchar música en streaming es la actividad más popular, ya que casi la mitad de los encuestados, un 48,2\%, declararon realizarla frecuentemente. Por el contrario, la asistencia a musicales se produce con menor intensidad, pues cerca del $80 \%$ de los individuos señaló no realizarla nunca. Asistir a conciertos en directo resulta ser la práctica más realizada de manera ocasional como señalaron casi la mitad, el 46,1\%, de los participantes en el estudio. Analizando las diferencias por géneros podemos comprobar que, si bien hombres y mujeres acuden a conciertos de música en vivo con similar proporción, independientemente del nivel de frecuencia, no ocurre lo mismo respecto a las otras actividades analizadas. Así, el porcentaje de hombres que escuchan música en streaming y la descargan de Internet es superior al de las mujeres. Por el contrario, a pesar de ser una actividad con bajo grado de realización en general, las mujeres asisten a musicales en mayor medida que los hombres. Con todo, tal y como demuestra la existencia de diferencias estadísticamente significativas, el género influye en la descarga de música, la escucha en streaming y la participación en musicales, pero no en la asistencia a conciertos de música en directo.

\section{CUADRO 4 \\ FRECUENCIA DE PARTICIPACIÓN EN ACTIVIDADES VINCULADAS CON LA MÚSICA}

(En \%)

\begin{tabular}{|c|c|c|c|c|c|}
\hline & & Nunca & $\begin{array}{c}\text { Ocasional- } \\
\text { mente }\end{array}$ & $\begin{array}{l}\text { Frecuente- } \\
\text { mente }\end{array}$ & $\chi^{2}$ (sig.) \\
\hline \multirow{3}{*}{$\begin{array}{l}\text { Descarga de música } \\
\text { de Internet }\end{array}$} & Total & 47,3 & 29,2 & 23,5 & - \\
\hline & Hombres & 41,7 & 33,8 & 24,5 & \multirow{2}{*}{$\begin{array}{l}\chi^{2}=10,81 \\
(p<0,01)\end{array}$} \\
\hline & Mujeres & 52,9 & 24,6 & 22,5 & \\
\hline \multirow{3}{*}{$\begin{array}{l}\text { Escucha de música } \\
\text { en streaming }\end{array}$} & Total & 31,3 & 20,5 & 48,2 & - \\
\hline & Hombres & 26,6 & 22,2 & 51,2 & \multirow{2}{*}{$\begin{array}{l}\chi^{2}=7,86 \\
(p<0,05)\end{array}$} \\
\hline & Mujeres & 36,1 & 18,7 & 45,2 & \\
\hline \multirow{3}{*}{$\begin{array}{l}\text { Asistencia a } \\
\text { musicales }\end{array}$} & Total & 79,3 & 18,2 & 2,5 & - \\
\hline & Hombres & 87,1 & 11,1 & 1,8 & \multirow{2}{*}{$\begin{array}{l}\chi^{2}=28,44 \\
(p<0,01)\end{array}$} \\
\hline & Mujeres & 71,4 & 25,4 & 3,2 & \\
\hline \multirow{3}{*}{$\begin{array}{c}\text { Asistencia a } \\
\text { conciertos en vivo }\end{array}$} & Total & 44,2 & 46,1 & 9,7 & - \\
\hline & Hombres & 44,1 & 46,2 & 9,7 & \multirow{2}{*}{$\begin{array}{l}\chi^{2}=0,009 \\
(p>0,05)\end{array}$} \\
\hline & Mujeres & 44,4 & 46,0 & 9,6 & \\
\hline
\end{tabular}

FUENTE: Elaboración propia. 


\subsection{Motivaciones de asistencia a conciertos}

Los participantes en el estudio valoraron once motivaciones relativas a la asistencia a conciertos de música en vivo. Las mismas, procedentes de la revisión de la literatura, fueron adaptadas al contexto de estudio. El Cuadro 5 muestra los valores medios de cada una de dichas motivaciones, así como también los valores medios según el género. Así, las principales razones para asistir a este tipo de eventos culturales son: gustarles la música $(4,47)$, querer ver a un artista o banda específicos $(4,37)$, y pasárselo bien $(4,37)$. Por el contrario, querer impresionar a alguien $(1,45) \mathrm{o}$ la búsqueda de horizontes más amplios $(2,43)$ son motivaciones poco valoradas por ambos sexos. El Gráfico 1 muestra la distribución de los diferentes motivos de manera detallada. En él se señala, para cada uno de los ítems, el porcentaje de individuos que los valoran positiva, negativa y neutralmente.

\section{CUADRO 5 \\ VALORES MEDIOS MOTIVACIONES DE ASISTENCIA A CONCIERTOS DE MÚSICA \\ (1: Total desacuerdo - 5: Total acuerdo)}

\begin{tabular}{|l|c|c|c|c|}
\hline \multicolumn{1}{|c|}{ Fui al concierto porque... } & Media global & Hombre & Mujer & Prueba $\boldsymbol{t}$ (sig.) \\
\hline me encanta la música & 4,47 & 4,46 & 4,47 & $\begin{array}{c}t=-0,027 \\
(p=0,98)\end{array}$ \\
\hline me invitó un amigo o familiar & 3,37 & 3,35 & 3,40 & $\begin{array}{c}t=-0,344 \\
(p=0,73)\end{array}$ \\
\hline quería ver a un artista/banda & 4,37 & 4,35 & 4,40 & $\begin{array}{l}t=-0,699 \\
(p=0,49)\end{array}$ \\
\hline alguien me lo dijo o recomendó & 3,22 & 3,27 & 3,16 & $\begin{array}{l}t=0,969 \\
(p=0,33)\end{array}$ \\
\hline me generaba curiosidad & 3,08 & 3,09 & 3,08 & $\begin{array}{c}t=0,105 \\
(p=0,92)\end{array}$ \\
\hline quería impresionar a alguien & 1,45 & 1,50 & 1,39 & $\begin{array}{c}t=1,484 \\
(p=0,14)\end{array}$ \\
\hline buscaba ampliar mis horizontes & 2,43 & 2,45 & 2,41 & $\begin{array}{c}t=0,271 \\
(p=0,79)\end{array}$ \\
\hline necesitaba desconectar & 2,72 & 2,67 & 2,78 & $\begin{array}{l}t=-0,812 \\
(p=0,42)\end{array}$ \\
\hline quería pasármelo bien & 4,37 & 4,32 & 4,42 & $\begin{array}{l}t=-1,329 \\
(p=0,18)\end{array}$ \\
\hline me apetecía socializar & 2,87 & 2,92 & 2,82 & $\begin{array}{c}t=0,894 \\
(p=0,37)\end{array}$ \\
\hline buscaba sentir emociones & 3,35 & 3,35 & 3,35 & $\begin{array}{l}t=0,013 \\
(p=0,99)\end{array}$ \\
\hline
\end{tabular}

FUENTE: Elaboración propia. 


\section{GRÁFICO 1 \\ REPRESENTACIÓN VALORACIÓN MOTIVOS DE ASISTENCIA A CONCIERTOS DE MÚSICA}

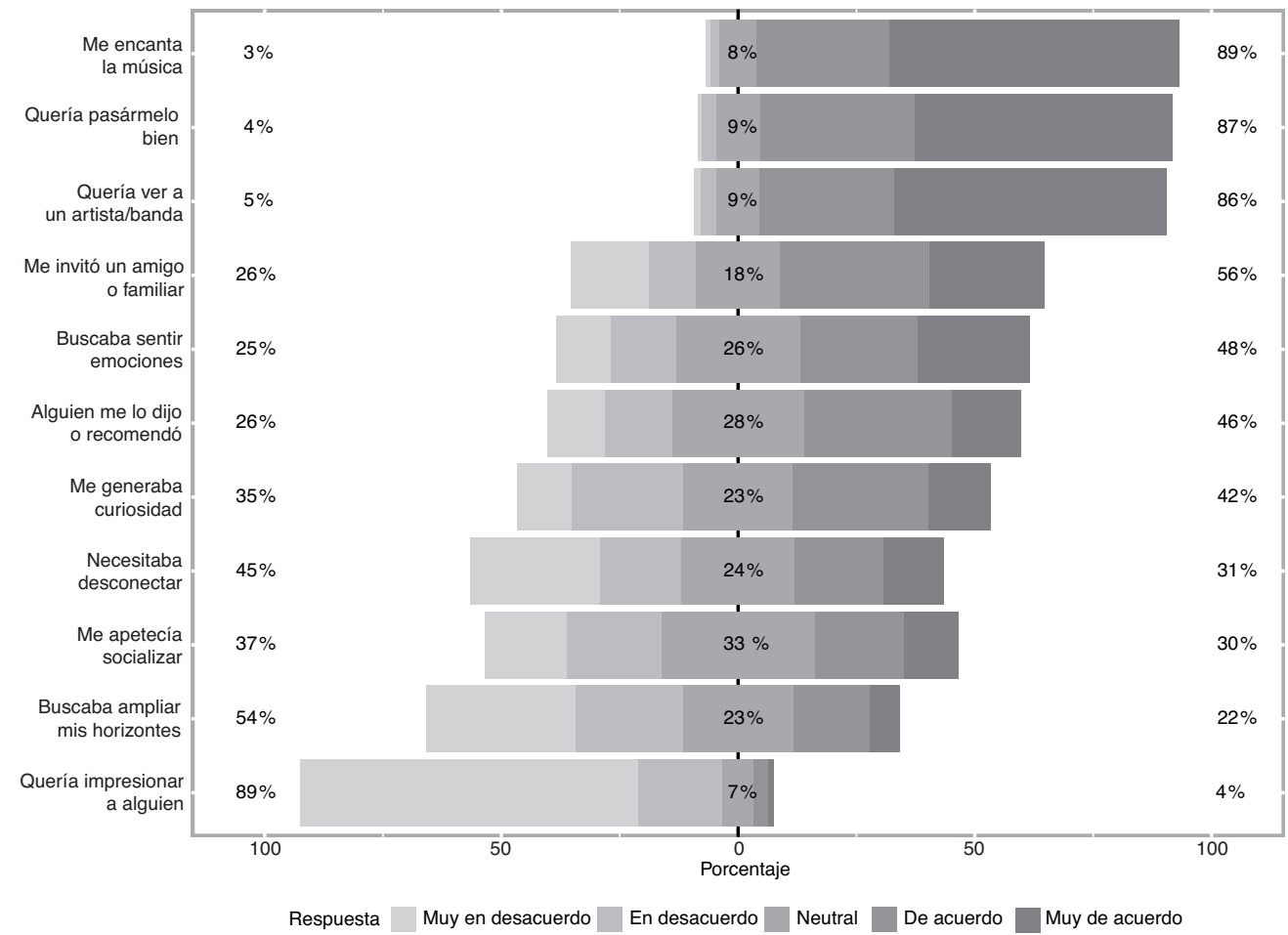

FUENTE: Elaboración propia.

El Cuadro 5 recoge también la valoración de las distintas motivaciones en función del género. En el mismo se pone de manifiesto la inexistencia de diferencias entre ambos grupos en relación a los motivos que impulsan a las personas a asistir a conciertos de música. El Gráfico 2 complementa esta información mostrando la distribución de las valoraciones para hombres y mujeres. En él se observa que tanto las principales razones de asistencia, como las que obtienen valoraciones intermedias o incluso las no relevantes se distribuyen de forma homogénea e independiente del género del encuestado. 


\section{GRÁFICO 2}

\section{REPRESENTACIÓN VALORACIÓN MOTIVOS DE ASISTENCIA A CONCIERTOS DE MÚSICA SEGÚN GÉNERO}

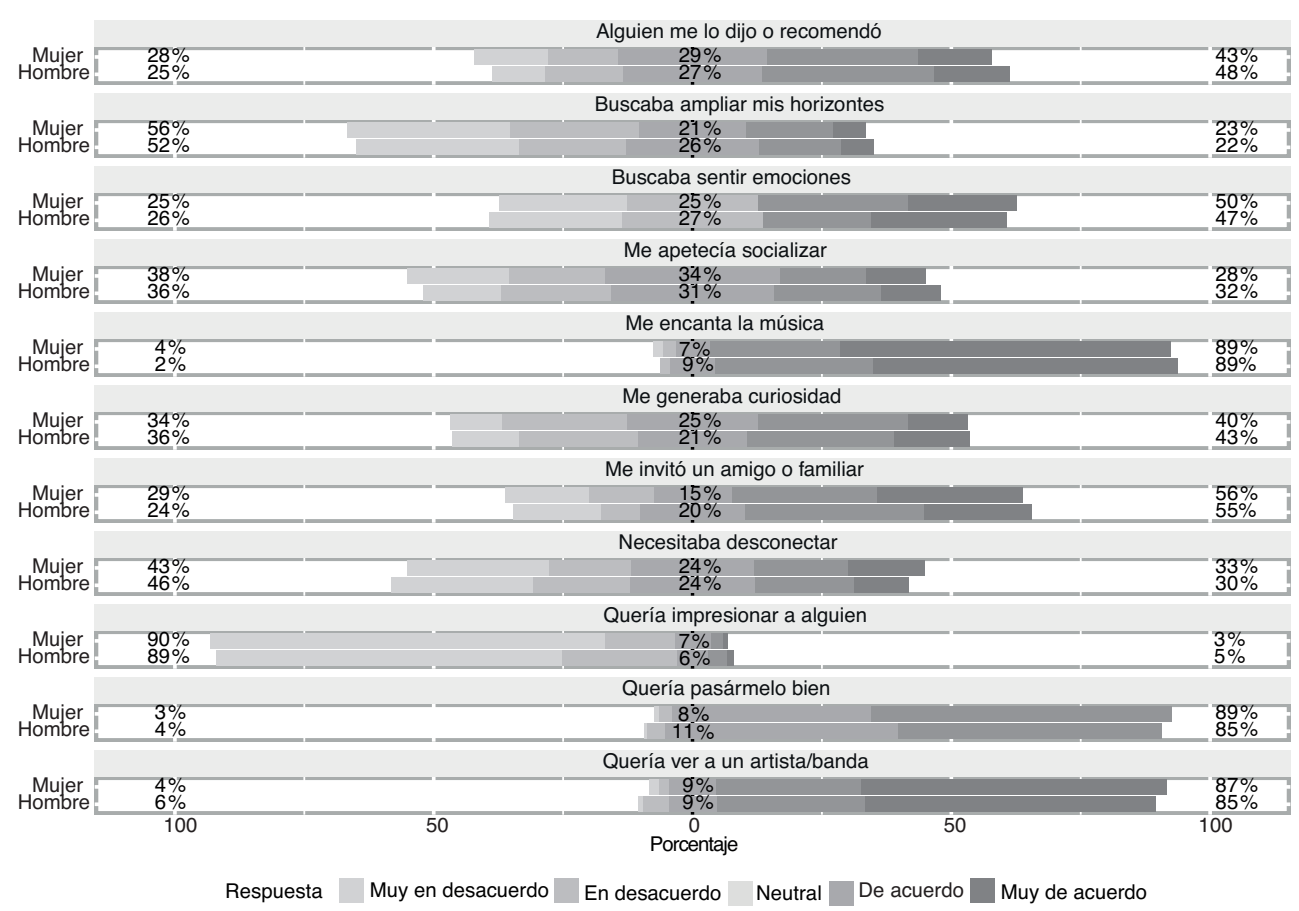

FUENTE: Elaboración propia.

\subsection{Frenos a la asistencia a conciertos}

Al igual que con los motivos, se solicitó a los individuos que valorasen una serie de ítems explicativos de los frenos a la asistencia a conciertos de música en vivo en el último año. Estos también fueron extraídos de estudios previos sobre barreras al consumo en el contexto cultural. El Cuadro 6 muestra los valores medios de cada uno de ellas para el total de la muestra, así como por géneros. En este caso, solo tres de estas barreras presentan una cierta relevancia frente al resto que muestran tener poco peso. Concretamente, los individuos manifiestan que el estar muy ocupado $(3,63)$, el elevado precio de las entradas $(3,29)$, y la preferencia por otras actividades de ocio $(3,11)$ limitan su asistencia. El Gráfico 3 aporta información visual sobre la distribución de todas las barreras consideradas y muestra como solo para las tres mencionadas hay un cierto consenso por parte de los encuestados (la frecuencia acumulada a la derecha del eje supera el $50 \%$ ). Contrariamente el resto acumulan la frecuencia a la izquierda, es decir, son irrelevantes para los individuos que forman la muestra. 


\section{CUADRO 6 \\ FRENOS A LA ASISTENCIA A CONCIERTOS DE MÚSICA \\ (1: Total desacuerdo - 5: Total acuerdo)}

\begin{tabular}{|l|c|c|c|c|}
\hline $\begin{array}{l}\text { No he asistido a ninguno o a } \\
\text { más conciertos porque... }\end{array}$ & Media global & Hombre & Mujer & Prueba $\boldsymbol{t}$ (sig.) \\
\hline $\begin{array}{l}\text { no me gusta especialmente la } \\
\text { música }\end{array}$ & 1,39 & 1,39 & 1,39 & $\begin{array}{c}t=-0,002 \\
(p=0,99)\end{array}$ \\
\hline $\begin{array}{l}\text { no iba a poder encontrar gente de } \\
\text { mi edad }\end{array}$ & 1,83 & 1,79 & 1,87 & $\begin{array}{c}t=-0,655 \\
(p=0,51)\end{array}$ \\
\hline estoy muy ocupado & 3,63 & 3,65 & 3,62 & $\begin{array}{c}t=0,181 \\
(p=0,86)\end{array}$ \\
\hline uno no puede moverse & 2,30 & 2,22 & 2,38 & $\begin{array}{l}t=-1,308 \\
(p=0,19)\end{array}$ \\
\hline no sé bien como disfrutar & 1,88 & 1,94 & 1,82 & $\begin{array}{c}t=1,236 \\
(p=0,22)\end{array}$ \\
\hline no tengo nadie con quien ir & 1,99 & 1,91 & 2,07 & $\begin{array}{c}t=-1,392 \\
(p=0,16)\end{array}$ \\
\hline las entradas cuestan mucho & 3,29 & 3,19 & 3,39 & $\begin{array}{l}t=-1,532 \\
(p=0,13)\end{array}$ \\
\hline $\begin{array}{l}\text { no se puede beber en los } \\
\text { conciertos }\end{array}$ & 1,70 & 1,90 & 1,51 & $\begin{array}{c}t=3,598 \\
(p=0,00)^{*}\end{array}$ \\
\hline $\begin{array}{l}\text { prefiero hacer otras actividades } \\
\text { de ocio }\end{array}$ & 3,11 & 3,08 & 3,14 & $\begin{array}{l}t=-0,414 \\
(p=0,68)\end{array}$ \\
\hline no merece la pena & 1,81 & 1,93 & 1,70 & $\begin{array}{c}t=2,349 \\
(p=0,02)^{*}\end{array}$ \\
\hline me sentiría incómodo & 1,87 & 1,95 & 1,80 & $\begin{array}{l}t=1,370 \\
(p=0,17)\end{array}$ \\
\hline
\end{tabular}

NOTA: $* p<0,05$.

FUENTE: Elaboración propia.

Sin embargo y a diferencia de los motivos de asistencia, en dos de los once frenos propuestos existen diferencias significativas entre hombres y mujeres. En concreto, estos son: el hecho de que no se pueda beber en los conciertos y que no les merezca la pena asistir. Ambos, poco relevantes para ambos géneros, son significativamente más valorados por los hombres que por las mujeres. En este sentido, no poder beber 


\section{GRÁFICO 3 \\ REPRESENTACIÓN VALORACIÓN FRENOS A LA ASISTENCIA A CONCIERTOS DE MÚSICA}

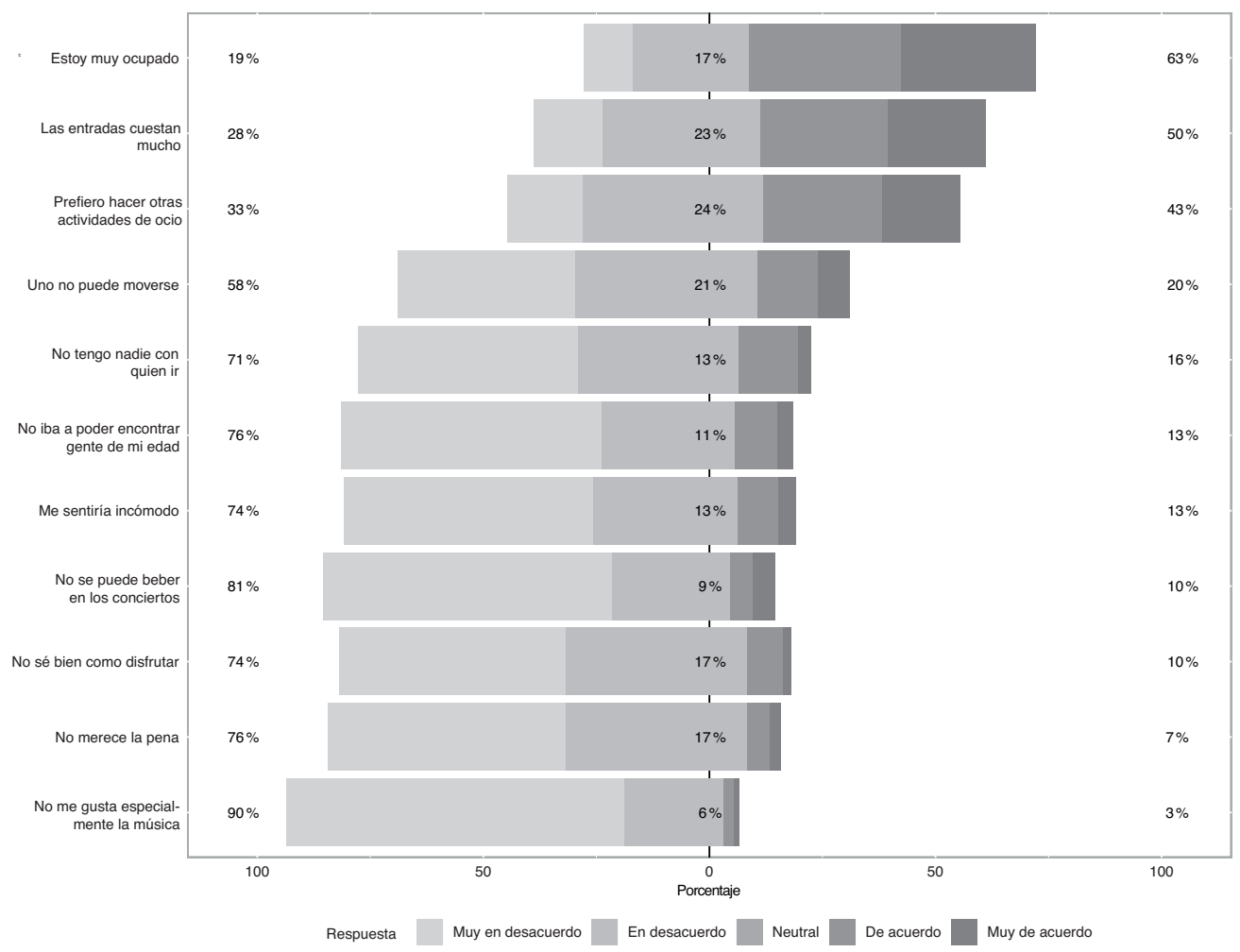

FUENTE: Elaboración propia.

alcanza una puntuación de 1,90 entre los hombres y de 1,51 entre las mujeres. La segunda barrera, que no merezca la pena asistir, obtiene una puntuación de 1,93 en el caso de los hombres y de 1,70 en el caso de las mujeres. Estos resultados, junto con el resto de frenos que no presentan diferencias significativas entre hombres y mujeres, se pueden visualizar en el Gráfico 4. 


\section{GRÁFICO 4 \\ REPRESENTACIÓN VALORACIÓN FRENOS A LA ASISTENCIA A CONCIERTOS DE MÚSICA SEGÚN GÉNERO}

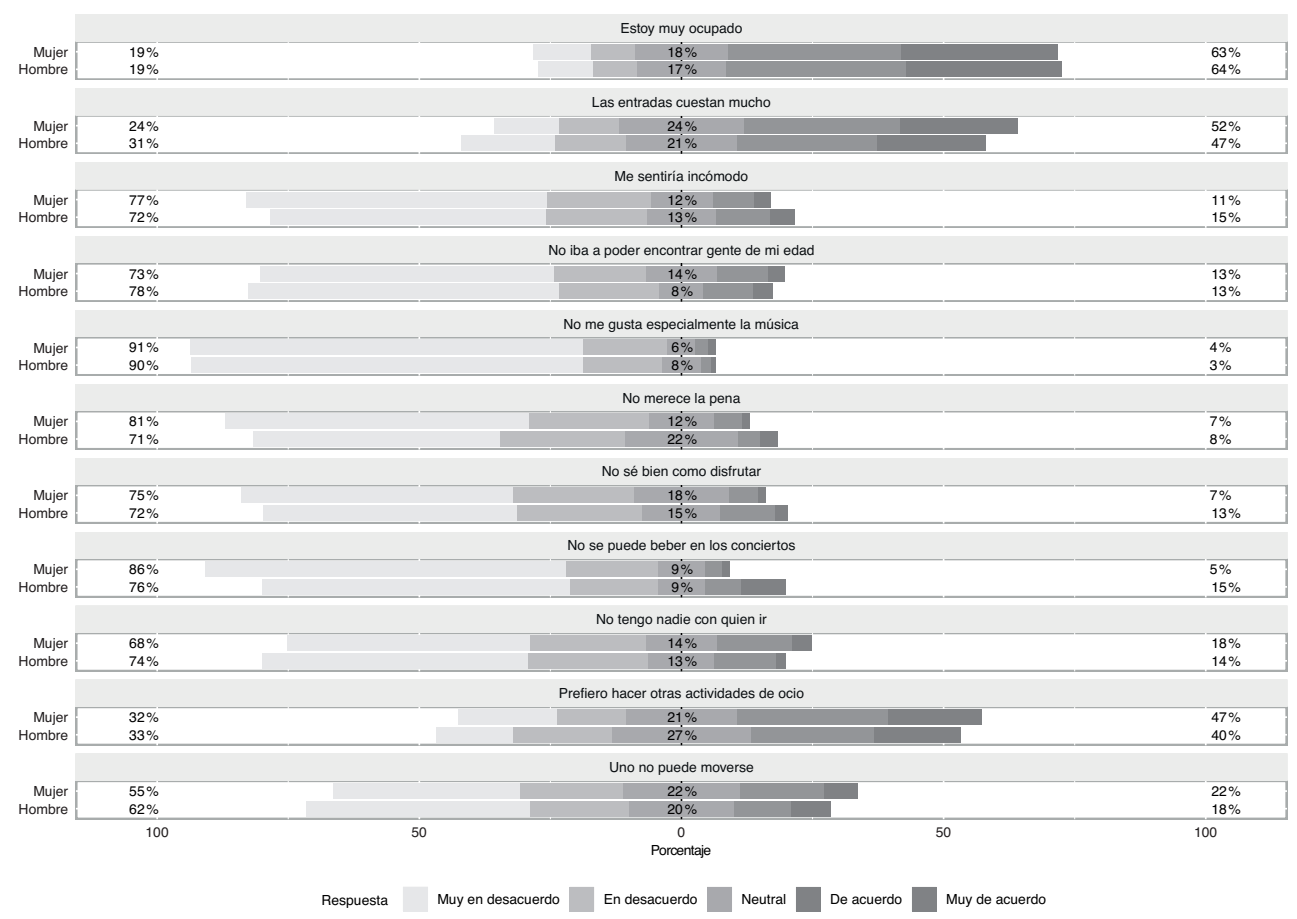

FUENTE: Elaboración propia.

\subsection{Predisposición a pagar por asistencia a conciertos}

Un último objetivo específico de este estudio perseguía conocer la predisposición a pagar por la entrada a un concierto de música. De los tres escenarios planteados: artista o banda local, nacional e internacional, ninguno presenta diferencias significativas para hombres y mujeres (Cuadro 7). Además, ambos sexos están dispuestos a pagar precios similares por una entrada a un concierto de música en vivo en función de la procedencia geográfica del artista o banda. En concreto, y considerando los porcentajes mayoritarios, los resultados señalan que el 54,2\% de los individuos muestra una disposición a pagar entre uno y 25 euros para ver a un artista o banda local; un 46,2\% entre 26 y 50 euros para un grupo nacional; y finalmente, el 55,8\% de los participantes señala la opción de estar dispuestos a pagar más de 50 euros para asistir a un concierto de un artista o banda internacional. 


\section{CUADRO 7}

PRECIO DISPUESTO A PAGAR POR ASISTENCIA A CONCIERTOS

(En \%)

\begin{tabular}{|c|c|c|c|c|c|}
\hline & & Total & Hombre & Mujer & \\
\hline \multirow{4}{*}{$\begin{array}{c}\text { Artista/banda } \\
\text { local }\end{array}$} & Nada & 20,1 & 21,4 & 18,7 & \multirow{4}{*}{$\begin{array}{l}X^{2}=1,36 \\
(p=0,71)\end{array}$} \\
\hline & $1-25 €$ & 54,2 & 52,2 & 56,1 & \\
\hline & $26-50 €$ & 19,7 & 20,3 & 19,1 & \\
\hline & Más de $50 €$ & 6,1 & 6,1 & 6,1 & \\
\hline \multirow{4}{*}{$\begin{array}{l}\text { Artista/banda } \\
\text { nacional }\end{array}$} & Nada & 3,7 & 3,4 & 4,0 & \multirow{4}{*}{$\begin{array}{l}X^{2}=3,86 \\
(p=0,28)\end{array}$} \\
\hline & $1-25 €$ & 32,1 & 32,7 & 31,6 & \\
\hline & $26-50 €$ & 46,2 & 43,6 & 48,9 & \\
\hline & Más de $50 €$ & 17,9 & 20,3 & 15,5 & \\
\hline \multirow{4}{*}{$\begin{array}{l}\text { Artista/banda } \\
\text { internacional }\end{array}$} & Nada & 1,9 & 1,8 & 1,9 & \multirow{4}{*}{$\begin{array}{l}X^{2}=1,68 \\
(p=0,64)\end{array}$} \\
\hline & $1-25$ euros & 9,2 & 8,4 & 9,8 & \\
\hline & 26-50 euros & 33,2 & 31,8 & 34,8 & \\
\hline & Más de $50 €$ & 55,8 & 58,0 & 53,5 & \\
\hline
\end{tabular}

FUENTE: Elaboración propia.

\section{Conclusiones}

La consideración del género es hoy día un tema de gran relevancia tanto a nivel social como académico. Su estudio en el ámbito de la gestión se ha centrado en conocer los aspectos que condicionan el comportamiento de los consumidores. En el contexto cultural son varias las contribuciones que han incluido esta variable. Concretamente, en el ámbito específico de la música algunos trabajos persiguen mostrar la existencia de diferencias de género en actitudes y comportamientos de consumo. Los resultados alcanzados por estos estudios de temáticas variadas no muestran resultados consistentes. Por ahondar en esta línea de trabajo desarrollamos una investigación descriptiva cuyo objetivo ha sido analizar la influencia del género en la asistencia a conciertos de música.

Los resultados ponen de manifiesto la no existencia de grandes diferencias entre hombres y mujeres en relación a la frecuencia de asistencia, las motivaciones y los frenos del consumo de música en directo. En esta línea, la EHPC 18-19 (Ministerio de Cultura y Deporte, 2019) mostró que el porcentaje de asistencia a espectáculos de música era ligeramente más elevado en hombres que en mujeres. Sin embargo, los resultados alcanzados sobre la muestra del presente estudio arrojan mayor similitud al no existir diferencias significativas en la frecuencia de asistencia según género. De igual modo, no encontramos diferencias entre hombres y mujeres en ninguna 
de las motivaciones a participar analizadas, pero sí en alguno de los frenos, coincidiendo con las conclusiones del estudio de Liao y Tsai (2018). Tampoco surgieron diferencias estadísticamente significativas entre las dos submuestras en cuanto a la predisposición a pagar por la entrada a un concierto, independientemente de la procedencia de los artistas.

Esta no diferencia del comportamiento analizado puede estar en consonancia con el trabajo etnográfico de Pielichaty (2015), quien estudió el papel del género en los espacios de un festival de rock y música popular. La autora sugiere que tales lugares son espacios liminales que pueden permitir a cualquiera de ambos géneros invertir las normas sociales y comportarse con abandono y libertad, lejos de las limitaciones de lo cotidiano. Sin embargo, constata que ni mujeres ni hombres se dejan llevar, creando así los eventos y festivales un microcosmos representativo del mundo social. Con todo, el estudio del género en el contexto de la música podría tomar en consideración otras variables vinculadas al campo de la socialización y los roles de género, variables que no se han incluido en este trabajo.

Finalmente, son varias las implicaciones que pueden derivarse de los resultados obtenidos. Por un lado, consideraciones de tipo social y de género, al producirse una paulatina disminución de la brecha entre hombres y mujeres en cuanto al consumo de un servicio cultural particular como es la música en vivo. Además, y desde el lado de la oferta, existen implicaciones de gestión que organizadores y promotores de conciertos y festivales podrían plantearse. Desde el diseño de la programación al de las campañas de comunicación con el objetivo de potenciar todo aquello que más valor pueda aportar a ambos colectivos y minorando el efecto de ciertas barreras de asistencia para así atraer y consolidar público independientemente de su género.

\section{Referencias bibliográficas}

Andersson, T., \& Carslen, J. (2011). Strategic SWOT analysis of public, private and nonfor-profit festival organisations. International Journal of Event and Festival Management, $2(1), 83-97$.

Birknerová, Z., Frankovský, M., Zbihlejová, L., \& Parová, V. (2018). Perception of Advertising and Expectations of Advertising in terms of Gender Differences. Marketing and Branding Research, 5, 92-99.

Carreño, T. (2015). Festivales en España. Estrategias generales de gestión. Intersección. Año 2, edición 3.

Chen, C. F., \& Hu, H. Y. (2006). The attitudes of university students to classical music concerts: A study in consumer behaviour. International Journal of Management, 23 (2), 366.

Colomer, J., \& Carreño, T. (2011). El paisaje de los festivales escénicos en España. En Bonet, L. \& Schargorodsky, H. (Dirs.), La gestión de festivales escénicos: conceptos, miradas y debates. Barcelona: Gescènic.

Connell, R. W. (2002). Gender. Cambridge: Polity Press.

Cviková, J., Debrecéniová, J., \& Kobová, L. (2007). Rodová rovnost'. Bratislava: Občan a demokracia [online]. Citado por Birknerová et al. (2018), op.cit. 
Eagly, A. H. (2013). Sex differences in social behavior: A social-role interpretation. Psychology Press.

Eckert, P., \& McConnell-Ginet, S. (2003). Language and gender. Cambridge: University Press.

Favaro, D., \& Frateschi, C. (2007). A discrete choice model of consumption of cultural goods: the case of music. Journal of Cultural Economics, 31 (3), 205-234.

Getz, D. (2010). The nature and scope of festival studies. International Journal of Event Management Research, 5 (1), 1-47.

Getz, D., \& Frisby, W. (1998). Evaluating Management effectiveness in community run festivals. Journal of Travel Research, Summer, 22-27.

Getz, D., Andersson, T., \& Larson, M. (2007). Festival stakeholder roles: concepts and case studies. Event Management, 10 (2/3), 103-122.

Gilmore, D. D. (1998). Carnival and Culture: Sex, Symbol, and Status in Spain. Yale University Press, New Haven, CT.

Hu, X., \& Lee, J. H. (2012). A cross-cultural study of music mood perception between American and Chinese listeners. Libro de actas de 13th International Society for Music Information Retrieval Conference (ISMIR), Porto, 535-540.

$\mathrm{Hu}, \mathrm{X} .$, \& Lee, J. H. (2016). Towards global music digital libraries: A cross-cultural comparison on the mood of Chinese music. Journal of Documentation, 72 (5), 858877.

Kellaris, J. J., \& Rice, R. C. (1993). The influence of tempo, loudness, and gender of listener on responses to music. Psychology \& Marketing, 10 (1), 15-29.

Kim, D. Y., Lehto, X.Y. \& Morrison, A. M. (2007). Gender differences in online travel information search: Implications for marketing communications on the internet. Tourism Management, 28 (2), 423-433.

Kinnally, W., Lacayo, A., McClung, S., \& Sapolsky, B. (2008). Getting up on the download: college students' motivations for acquiring music via the web. New Media \& Society, 10 (6), 893-913.

Krook, M. L., \& Childs, S. (2010). Women, gender and politics. Oxford: Oxford University Press.

Krueger, A. B. (2005). The economics of real superstars: The market for rock concerts in the material world. Journal of Labor Economics, 23 (1), 1-30.

Lee, J. H. \& Hu, X. (2014). Cross-cultural similarities and differences in music mood perception. Libro de actas de iConference, 259-269.

Liao, H. L., \& Tsai, H. Y. (2018). Understanding attitudes towards music leisure activity and the constraints faced by the elderly. International Journal of Organizational Innovation, 11 (2), 160-173.

Meyers-Levy, J., \& Maheswaran, D. (1991). Exploring differences in males' and females' processing strategies. Journal of Consumer Research, 18 (1), 63-70.

Ministerio de Cultura y Deporte (2019). Encuesta de Hábitos y Prácticas Culturales 20182019.

Montoro-Pons, J. D., \& Cuadrado-García, M. (2011). Live and prerecorded popular music consumption. Journal of Cultural Economics, 35 (1), 19-48.

Montoro-Pons, J. D., \& Cuadrado-García, M. (2016). Unveiling Latent Demand in the Cultural Industries: An Application to Live Music Participation. International Journal of Arts Management, 18 (3), 5-24. 
Nel, J., Raubenheimer, J., \& Bounagui, M. (2009). Gender differences in purchase intention of music downloads. Management Dynamics: Journal of the Southern African Institute for Management Scientists, 18 (3), 25-36.

Oakley, A. (1972). Sex, gender and society. London: Maurice Temple Smith Ltd.

Pielichaty, H. (2015). Festival space: gender, liminality and the carnivalesque. International Journal of Event and Festival Management, 6 (3), 235-250.

Prieto-Rodriguez, J., \& Fernandez-Blanco, V. (2000). Are popular and classical music listeners the same people? Journal of Cultural Economics, 24 (2), 147-164.

Putrevu, S. (2001). Exploring the origins and information processing differences between men and women: Implications for advertisers. Academy of Marketing Science Review, 10 (1), 1-14.

Richards, G. (2007). The festivalization of society or the socialization of festivals? The case of Catalunya. En Richards, G. (Ed.), Cultural tourism: global and local perspectives. Binghampton: Haworth Hospitality Press.

Sociedad General de Autores y Editores (2018). Anuario de las artes escénicas, musicales y audiovisuales. Fundación SGAE. Madrid. Recuperado de http://www.anuariossgae.com/ anuario2018/home.html. Consultado el 16 de julio de 2019.

Suhányi, L., \& Suhányiová, A. (2014). Multi-criteria decision-making tool design for the investment decision-making of territorial self-government regions. Journal of applied economic sciences, 9 (1), 110-122.

Van Eijck, K. (2001). Social differentiation in musical taste patterns. Social Forces, 79 (3), 1163-1185.

Webster, G. D., \& Weir, C. G. (2005). Emotional responses to music: Interactive effects of mode, texture, and tempo. Motivation and Emotion, 29 (1), 19-39. 